\title{
IMPORTANCE OF INVOLVING PATIENTS AND PUBLIC IN HEALTH RESEARCH IN BANGLADESH AND NEPAL
}

*Corresponding Author: Dr Bibha Simkhada

*1. Dr Bibha Simkhada (RN, BA, BSc, PhD), Senior Lecturer in Adult Nursing, Department of Nursing and Midwifery, School of Human and Health Sciences, University of Huddersfield, Queensgate, Huddersfield, HD1 3DH Huddersfield, UK. Email:

b.d.simkhada@hud.ac.uk

2. Prof Edwin van Teijlingen (MA, MEd, PhD), Professor of Reproductive Health Research, CMMPH, Bournemouth University, UK.

3. Dr Aliya Naheed (MBBS, MPH, PhD), Head, Initiative for Non-communicable Diseases, Health systems and Population Studies Division, icddr,b Bangladesh.

4. Angela Warren [BEd (Hons)], Carer \& Service User Co-ordinator: BU PIER Partnership, Faculty of Health \& Social Sciences, Bournemouth University, UK

5. Dr Sue Green, (RN, BSc, MMedSci, PhD), Principle Academic and Interim Deputy Head of Department for Nursing Science, Faculty of Health and Social Sciences, Bournemouth University 


\title{
IMPORTANCE OF INVOLVING PATIENTS AND PUBLIC IN HEALTH RESEARCH IN BANGLADESH AND NEPAL
}

\begin{abstract}
Patient and Public Involvement/Engagement (PPI/E) in public health research and Health Technology Assessment (HTA) in High-Income Countries (HIC) has significantly increased over past decade. PPI/E helps improve research and HTA ultimately benefitting patients and service users.

$\mathrm{PPI} / \mathrm{E}$ is very new concept in many LMICs (Low- and Middle-Income Countries). This paper considers the importance of PPI in public health research and HTA in the development and implementation of technology in the health sector in South Asia. Currently, in this region, health technology is frequently adopted from HICs without local research and HTA. It also discusses the importance of local co-creation of technology to reflect the needs of users within a culturally appropriate setting. It is important for LMIC-based researchers to understand the potential of PPI/E and how it can contribute to it to improve health care and research, especially perhaps in the era of COVID-19.
\end{abstract}

Keywords: Health Technology Assessment, Patient Public Involvement/Engagement (PPI/E), South Asia, LMIC.

Short (running) title: Patient and public involvement in health research 


\section{Background}

Patient and Public Involvement/Engagement (PPI/E) is vital in research and Health Technology Assessment (HTA) supporting a patient/person-centred care approach. There is a growing interest in implementing PPI/E in health research in LMIC ((Low- and Middle-Income Countries) because it benefits the: (a) research question; (b) conduct of the research; and (c) beneficiaries $(1,2)$. The UK organisation INVOLVE (3) defines public involvement as "research being carried out 'with' or 'by' members of the public rather than 'to', 'about' or 'for' them”. Public can refer to patients, potential patients, carers and people from organizations that represent service users as well as members of the public. Thus PPI 'occurs when individuals meaningfully and actively collaborate in the governance, priority setting, and conduct of research, as well as in summarizing, distributing, sharing, and applying its resulting knowledge' (4).

HTA is a systematic approach to evaluate the properties, effects, and impacts of health technologies or interventions (5), to inform policy and clinical decision making in the application of health technologies. HTA involves interdisciplinary research to assess cost effectiveness, budget impact, programmatic feasibility and social and ethical issues of health interventions (6). Health technology is critical elements in Universal Health Coverage (UHC) (7) and HTA is recognised as key to securing UHC through the efficient and equitable allocation of health care and resources (8). Therefore, promoting patient and public perspectives on the technologies being used is essential to assess the overall societal impact of health technologies. Integrating the patient perspective in public health research and HTA activities has the potential to improve the quality of healthcare services through meaningful contributions from and to the patient and public.

PPI/E could be used in South Asia, considering, as Cook and colleagues (1) describe, 'the variations in research infrastructure, cultural differences, the power differential between 
researched and researcher and often, lower research budgets.' Similarly, the growing use of technology in South Asia could be co-created locally to meet the literacy needs of users.

\section{Need for PPI/E}

One aspect of PPI/E is advising on and writing lay summaries; describing the research in language that ordinary people can understand and is relevant to their context. Involving people with local knowledge and experience of the area of research not only increases the potential outcome of the research but also achieve academic, economic and societal impact in the wider community (9).

PPI can occur in specific or all phases of the research cycle, from prioritising research area, research design, assisting with recruitment of study participants, data collection and analysis, monitoring and evaluation to dissemination $(10,11)$. Starting with developing the research proposal, PPI/E could start with preliminary discussions to identify research priorities. During the research process: PPI/E could help assess the research instrument being used such as the appropriateness, wording, language and timing of research instruments (e.g. questionnaires, interview schedules) to the public. They can be consulted to see whether they understand or interpret the data/findings in the same way as research team. At the dissemination stage people could help to develop patient information leaflets/websites or other research materials for awareness-raising of the research through various media.

\section{What and whom to ask}

In PPI/E as in any part of research and HTA you need to make sure you ask the right kind of questions for the right reasons and technology being tested. In other words: "What are you asking, and why are you asking it?" Also you need to consider which sub-group in the population represents your PPI best. 
Some see PPI/E as a burden, perhaps as another obstacle in research in a generally risk-averse society. Others, more positive towards PPI/E, fear an element of tokenism, the rise of the socalled professional patient. Examples of that can be seen in the grant application process in the UK, where the same paid member of staff of a health charity appears as PPI/E representative on several competing grants.

\section{PPI/E in South Asia}

There has been limited application of PPI/E in the more traditional patriarchal countries of South Asia. PPI is less patriarchal and more democratic, as it gives people a voice in shaping services, decision-making and research. The latter can be a threat to highly trained researchers (as PPI was for some in Europe 25 years ago), who may fail to see the value of the 'lay person's' voice or fear that involving patients may include, what may be deemed, irrelevant remark, only offers a few unrepresentative perspectives and threaten the scientific rigour of their research, which could make the research unfeasible (12).

We have been researching health in South Asia, mainly Nepal and Bangladesh for 15 years, but we have only recently started make users part of the research process early on. PPI/E is not common in the health research landscape in South Asia. A recent systematic review on health research in LMICs considered involvement and impact (1) found 62 studies and PPI was mostly used in the planning stage and collaborations were the most common level of involvement. The review found little evidence of effectiveness and impact tended to relate to increased relevance to the community, empowerment of participants and alterations in study design. Cook and colleagues' (1) overall conclusion was that proper PPI involvement in health research in LMICs is still rare. 


\section{Nepal}

We are involving users to develop nursing Continuous Professional Development (CPD). Nurses provided the PPI/E part of the research proposal development. PPI/E has helped us to understand the need of nurses however we still feel we have failed to recognise the patient and public perspective. We planned the recommendations with PPI/E input around dissemination events with nurse practitioners and policy makers in Kathmandu to achieve acceptance by national nursing/health policy-makers. We shared what works and what is feasible in CPD to enhance nurses' knowledge and skills. The nurses valued their involvement in dissemination event and reported it provided them with ownership in the CPD framework development. We now realised that involving patients could have given us insight in their perceptions of CPD training from the research proposal stage i.e. how it can benefit nurse-patient interactions, for example to help nurses understanding patients' perspectives in the CPD training. The main idea of CPD is to offers the opportunity to maintain, improve and broaden knowledge, expertise and enhance nurses' practice and improve nurse's interpersonal relationships with multidisciplinary team, patients and family for better quality of care $(13,14)$

\section{Bangladesh}

A multi component hypertension control programme in Bangladesh driven by rural Community Health Workers (CHWs) in collaborations with government doctors has set an example of the value of PPE in strengthening primary health systems for delivery of hypertension (15). A group of researchers in Bangladesh, Pakistan, Sri Lanka and Singapore evaluated the impact of a multi component hypertension control program titled 'Control of Blood Pressure and Risk Attenuation in Bangladesh, Pakistan, and Sri Lanka (COBRA-BPS) (16). The CHWs were trained to use a digital blood pressure monitor (Omron: HEM-7300) to measure blood pressure of the hypertensive study participants during routine home. The CHWs also conducted home-based health education with these patients and their family to modify 
lifestyle as well as promote medication adherence. The CHWs in Bangladesh detected patients with uncontrolled blood pressure and mobilized the family to refer them to government primary health facilities for management of uncontrolled blood pressure by trained doctors. A significance reduction of blood pressure was observed among the patients in the intervention areas compared to those in the usual care after two years (2016-2018).

The proactive engagement of the CHWs with the patients in the community and primary-care doctors created a high demand for hypertension care and subsequently anti-hypertensive medicines were made available at the primary health facilities throughout the country. PPI/E was a key part of the COBRA-BPS programme's implementation, and has demonstrated the value of involving PPI/E in improving the quality of care as well as acceptability of health interventions in low resource settings in South Asia.

\section{Developing PPI/E in a culturally appropriate way}

In South Asia health workers are often seen as expert in patients' conditions. It is important for researcher to understand this context. Therefore it is important to consider practical questions when planning for PPI/E i.e. how do we change this culture? How do we empower patients to see that they have a very unique expertise of their own building rapport and creating opportunities for collaboration takes time (and should be factored/costed into any bids). How and where do we create an environment where people feel safe and it is familiar to them? Are there barriers to break through where only 'community leaders' are consulted? One key way to involve people is to 'go to where they are,' but will there be issues of trust - unless of course, there is someone from that culture/community as part of the research team or the people themselves are trained as researchers.)

Cultural influences, politics, and economic status can play a big role in South Asia, and as such would affect PPI/E in public health and HTA research. There is increasing interest in human- 
centric design in the public health and HTA research to test locally the new technology adopted from HICs. In Nepal and Bangladesh health workers are known as the experts; they know 'everything' about the patient's condition and the patient follow what is being said; having little/no voice in their own health care planning. Therefore it is important to explore how PPI/E could effectively work in a South Asian context and ascertain whether people value being involved in research design, processes and dissemination.

Challenges of PPI/E may be avoided through clear planning of the PPI/E activity in the earlyplanning stages of the proposed study (17) and more importantly there is need for awareness on importance of the patient involvement in the research in South Asia. Researcher also need to understand the users feeling of empowered, valued, listened to and generally more positive about their experiences when they engages in the research process (18). When researchers planned PPI/E it is important to communicate in a culturally appropriate way, educate the public that their views are important and useful in the service provision. However, creating a mechanism for accountability of the health care providers to patients and public would remain a challenge as well as an essential element for promoting PPI/E in South Asian settings. The patients and the public can provide valuable input; offering a unique perspective in the project. In Nepal and Bangladesh, there is lay involvement in the process of dissemination, often with stakeholders (service providers) however there is some notion of community engagement in health education/promotion programmes. There may be a highly educated representative of the community acting as the voice of the people, for example inclusion of different ethnicity, rural and urban and gender.

It is important to have representative and diverse people in the group to avoid the overemphasizing of particular problems; groups being dominated by strong characters and their perspectives; groups being overshadowed by personal experience stories. Representation of the population is not only the element of the PPI, it is important they have some kind of decision 
making around research process. They should have key role on dissemination of research findings, as they are able to provide access to a wider audience than traditional academic circles. We believe PPI/E can improve the quality of research projects, particular developing health interventions and strengthen their relevance and increasing the likelihood of achieving impact. The global pandemic COVID-19 also adds the value of PPI/E in designing research grants or surveys in Nepal and Bangladesh including some South Asian countries as there are some similarities in their cultural practice and health research environments.

"Conflicts of Interest: None".

"Funding Statement: This research received no specific grant from any funding agency, commercial or not-for-profit sectors." 


\section{References}

1. Cook N, Siddiqi N, Twiddy M and Kenyon R. Patient and public involvement in health research in low and middle-income countries: a systematic review, BMJ Open. 2019; 9:e026514. doi: 10.1136/bmjopen-2018-026514

2. Puerta LL, Bartlam B and Smith H. Researchers' perspectives on public involvement in health research in Singapore: The argument for a community-based approach. Health Expectations. 2019; 22:666-675.

3. NIHR/INVOLVE. What is public involvement in research? 2019. Available in [https://www.invo.org.uk/find-out-more/what-is-public-involvement-in-research-2/]. Accessed on Nov 2019.

4. IHRF. Public and Patient Involvement (PPI) in Research. Irish Health Research Forum. 2015. Available in [https://859556ce-4d33-4b82-be63-d48940ba7029.filesusr.com/ugd/ 75eae6_5ee17a6102544c3c8119362d36bb751b.pdf]. Accessed on Feb 2020.

5. WHO. 2015 Global Survey on Health Technology Assessment by National Authorities. 2017. Available in [https://www.who.int/health-technology-assessment/MD_HTA_oct2015_ final_web2.pdf?ua=1]. Accessed on Nov 2019.

6. Hailey D, Babidge W, Cameron A and Davignon LA. HTA agencies and decision makers: An INAHTA guidance document. INAHTA. 2010. Available in [http://www.inahta.org/wpcontent/themes/inahta/img/HTA\%20\%20Decision\%20Makers.pdf]. Accessed on Feb 2020.

7. WHO. Universal health coverage (UHC). 2019. Available in [https://www.who.int/newsroom/fact-sheets/detail/universal-health-coverage-(uhc)]. Accessed on April 2020. 
8. Chalkidou K, Marten R, Cutler D, Culyer T, Smith R, Teerawattananon Y, et al. Health technology assessment in universal health coverage. Lancet. 2013; 382(9910): e48-e49.

9.Hughes M and Duffy C. Public involvement in health and social sciences research: A concept analysis. Health Expectations. 2018; 21:10.1111/hex.12825.

10. Greenhalgh T, Hinton L, Finlay T, Macfarlane A, Fahy N, Clyde B et al. Frameworks for supporting patient and public involvement in research: Systematic review and co-design pilot. Health expectation. 2019; 22:785-801.

11. Ball S, Harshfield A, Carpenter A, Bertscher A and Marjanovic S. Patient and public involvement and engagement in research enabling meaningful contributions. RAND Corporation. 2019. Available on [https://www.rand.org/pubs/research_reports/RR2678.html]. Accessed on Jan 2020.

12. Turk A, Boylan AM and Locock L A. Researcher's Guide to Patient and Public Involvement. 2017. Available in [https://oxfordbrc.nihr.ac.uk/wp-content/uploads/2017/03/AResearchers-Guide-to-PPI.pdf]. Accessed on March 2020.

13. RCN. RCN Factsheet: Continuing Professional Development (CPD) for nurses working in the United Kingdom, Royal college of Nursing UK. 2016. Available in [https:// www.rcn.org.uk/about-us/policy-briefings/ pol-1614]. Accessed on Jan 2020

14. Simkhada B, Mackay S, Khatri R, Sharma CK, Pokhrel T, Marahatta S, et al. Continual Professional Development (CPD): Improving Quality of Nursing Care in Nepal. Health Prospect: Journal of Public Health. 2016; 15(3).

15. Jafar TH, Jehan I, de Silva HA, Naheed A, Gandhi M, Assam P, et al. Multicomponent intervention versus usual care for management of hypertension in rural Bangladesh, Pakistan 
and Sri Lanka: study protocol for a cluster randomized controlled trial. Trials, 2017. 18(1): p. 272.

16. Jafar TH, Gandhi M, de Silva HA, Jehan I, Naheed A, Finkelstein EA, et al. A Community-Based Intervention for Managing Hypertension in Rural South Asia. New England Journal of Medicine. 2020; 382(8):717-726.

17. Brett J, Staniszewska S, Mockford C, Herron-Marx S, Hughes J, Tysall C et al. Mapping the impact of patient and public involvement on health and social care research- a systematic review. Health Expectations. 2012; 17:637-650.

18. Brett J, Staniszewska S, Mockford C, Herron-Marx S, Hughes J, Tysall C et al. A Systematic Review of the Impact of Patient and Public Involvement on Service Users, Researchers and Communities. Patient. 2014; 7:387-395. 